\title{
Diseño de objetos de aprendizaje para comunicación, mercadeo y artes
}

\section{Design of Communication, Marketing, and Arts Learning Objects}

\author{
Johnny Waldo López Jerez \\ Magister en Tecnología Educativa \\ johnny.lopez@uniminuto.edu \\ Corporación Universitaria Minuto de Dios
}

\section{Resumen \\ El presente artículo pretende establecer, desde una perspectiva educomunicativa, algunos factores que intervienen en el análisis del diseño instruccional de los objetos de aprendizaje para programas académicos en las áreas del mercadeo, la comunicación y las artes. Busca determinar los elementos de la teoría constructivista y el enfoque de competencias que se podrían implementar en el diseño de este tipo particular de materiales educativos digitales y establecer lineamientos sobre las didácticas propias de este}

\begin{abstract}
This article aims to establish, from an educommunicative perspective, some factors that take part in the analysis of the instructional design of learning objects for academic programs in the areas of marketing, communication, and arts. This article claims to define elements of the Constructivist Theory and of the competence approach that could be implemented when designing this particular type of digital learning material. It also suggests guidelines for the teaching of this type of programs, especially at
\end{abstract}

Para citar este artículo / to CIte this ARticle López Jerez, J. W. (2014). Diseño de objetos de aprendizaje para comunicación, mercadeo y artes. Poliantea, 10119), pp. 215-233. 
- Diseño de objetos de aprendizaje para comunicación - Johnny Waldo López Jerez

tipo de programas en el caso puntual de la Institución Universitaria Politécnico Grancolombiano de Bogotá (Colombia).

Palabras clave: objeto de aprendizaje, diseño instruccional, competencias.
Politécnico Grancolombiano University Institution in Bogotá, Colombia.

Keywords: Learning object, teaching design, competences. 


\title{
Diseño de objetos de aprendizaje para comunicación, mercadeo y $\operatorname{artes}^{1}$
}

\section{Design of Communication, Marketing, and Arts Learning Objects}

\author{
Johnny Waldo López Jerez \\ Magíster en Tecnología Educativa \\ Corporación Universitaria Minuto de Dios
}

\section{Introducción}

La presente investigación se desarrolló durante 2011 en el contexto de la Facultad de Mercadeo, Comunicación y Artes de la Institución Universitaria Politécnico Grancolombiano, cuya sede principal se encuentra en Bogotá (Colombia) y que imparte programas académicos tanto en la modalidad presencial como virtual. Desde el punto de vista educativo, pretende orientar el diseño de sus currículos desde un enfoque de diseño por competencias profesionales, bajo el sistema de créditos académicos, organizando los programas en bloques de formación o áreas de profundización y, en algunos casos, diseñarlos por ciclos propedéuticos. Adicionalmente, a través de los diversos cursos busca promover el autoestudio y la autonomía intelectual, así como estimular la formación en valores, emprendimiento, apropiación de la tecnología y promoción de la cultura ambiental y el desarrollo sostenible.

Es importante recalcar que el Politécnico Grancolombiano asume como política educativa un uso generalizado y racional de las tecnologías de la 
información y la comunicación como apoyo a la educación, como oportunidad para brindar opciones de flexibilidad y promover la autonomía en la formación de sus estudiantes.

No obstante, pese a que el movimiento del diseño de materiales educativos digitales se ha extendido en el mundo como una prioridad para la educación del siglo XXI, y que en Colombia se han adelantado proyectos tendientes al desarrollo de iniciativas gubernamentales y universitarias para trabajar en el tema, el Politécnico Grancolombiano aún no cuenta con un programa permanente en el área que le haya permitido alinear este tema como parte de su estrategia educativa, aunque desde luego existe un gran interés en que se inicie el proceso, pero buscando coherencia con las necesidades reales de los programas académicos y las posibilidades tecnológicas con que cuenta la institución.

Este contexto es el que lleva a plantear la necesidad de indagar cuáles serían los principios didácticos con los que se deberían diseñar los recursos educativos digitales para la Facultad, con el fin de que su diseño, además de cumplir con las características tecnológicas necesarias para ser recuperables, usables, reutilizables, escalables e interoperables, también sean pertinentes y efectivos en la formación en conceptos y destrezas puntuales, propios de los programas involucrados.

Para dicho fin, se plantea entonces como objetivo principal "determinar las características educomunicativas involucradas en el diseño instruccional de objetos de aprendizaje (OA) para los programas académicos presenciales de la facultad", en el entendido de que, si bien hay un desarrollo conceptual respecto de las características instruccionales para el diseño de este tipo de recursos educativos digitales, no existe un volumen importante de documentación que permita orientar su creación desde parámetros didácticos propios de las ciencias de la comunicación, el mercadeo, la publicidad y el diseño; áreas de conocimiento en las que específicamente se enfoca la facultad.

Desde esta perspectiva, es bien conocido el desarrollo teórico que desde el punto de vista tecnológico hay respecto al concepto de objetos de aprendizaje, pero también la dificultad para encontrar criterios didácticos claros que permitan orientar su diseño en lo puramente educativo, más aún para diseños didácticos particulares de algunas disciplinas. Lo 
que ha generado la falsa ilusión de que todo diseño general es aplicable a las particularidades disciplinares y a las necesidades de formación de las distintas áreas de conocimiento. Así, la pretensión de esta investigación, más que formular modelos, era contar un conjunto de criterios de diseño instrucional para el diseño de objetos de aprendizaje coherentes con las áreas de conocimiento propias de los programas de la Facultad.

\section{Metodología}

Con el fin de determinar las características educomunicativas más adecuadas para el diseño de objetos de aprendizaje para los programas académicos de la Facultad, se planteó un diseño de investigación dividido en dos fases: una primera etapa exploratorio-documental sobre teorías de enseñanza-aprendizaje, estrategias pedagógicas y técnicas didácticas en el diseño general de recursos educativos, y en particular en los orientados a los programas de la Facultad. Una segunda etapa cuantitativo-descriptiva que indagará hábitos de uso, grado de satisfacción, percepciones y tipología de recursos educativos digitales implementados por los docentes de la Facultad.
Para la primera etapa se consultó una gran cantidad de información respecto del diseño de materiales educativos digitales y, en especial, sobre el diseño de objetos de aprendizaje, lo que permitió construir una base teórica sobre este concepto que podría adoptar la institución y las características técnicas implicadas, así como diversas teorías sobre el denominado diseño instruccional, lo que permitió acercarse a los principios educomunicativos que deberían tener presentes objetos de aprendizaje que estuvieran sustentados en pedagogías activas y basados en un enfoque de competencias, características que son inherentes al modelo educativo de la institución.

Para la segunda etapa se adelantó una encuesta entre los docentes de la Facultad con el fin de conocer el conocimiento, el uso y las percepciones sobre las tecnologías de la información y la comunicación como apoyo a sus cátedras, y por otro lado se hizo una revisión de la forma de uso y tipo de recursos digitales usados en la plataforma de educación virtual de la institución y en la que todos los docentes tienen aulas virtuales asignadas, aunque no siempre son usadas de manera intensiva.

Respecto de la encuesta, se estimó que para una población de 177 
profesores que impartían cursos en modalidad presencial en la Facultad, la muestra debía ser de 122 encuestas, teniendo en cuenta un margen de error de $5 \%$ y un nivel de confianza esperado de $95 \%$. Mientras que para el análisis de aulas virtuales, teniendo que había 790 (para el primer semestre de 2011416 aulas y para el segundo 374 aulas), la muestra debía ser de 259 aulas, para un margen de error de $5 \%$ y un nivel de confianza esperado de $95 \%$. Estas aulas se distribuyeron para su análisis equitativamente según la cantidad total que había para cada programa académico, de modo que el muestreo fuera representativo del total de aulas de la Facultad y del porcentaje que representaba para cada programa.

\section{Marco conceptual}

Con el surgimiento y continuo desarrollo de internet se abrió paso rápidamente la idea de que fuera usado como medio, espacio o recurso para el aprendizaje. Desde diversos campos del saber se ha venido configurando un corpus teórico y experiencial sobre lo que puede hacerse con esta tecnología en beneficio de la educación. Uno de los movimientos más entusiastas es el de los denominados objetos de aprendizaje, especie de mediadores tecnológicos que permitirían enseñar cualquier cosa mediante el uso de piezas digitales distribuidas a través de redes.

Esta idea, la de construir piezas digitales para enseñar, no es nueva y tiene antecedentes que se remontan a la década de 1960. En el contexto de la instrucción basada en computador, Gerard (1969), citado en Gibbons, Nelson y Richards (2000), describía este tipo de objetos como "pequeñas unidades curriculares que pueden ser combinadas, como las piezas estandarizadas de un juego de Meccano, en una gran variedad de programas específicos hechos a la medida de cada estudiante".

Más tarde serían Atkinson y Wilson (1969) quienes trabajarían más profundamente sobre tres características inherentes a estos dispositivos: adaptabilidad, generatividad y escalabilidad. Se imponían y desarrollaban con esto principios que hasta hoy día se encuentran presentes en la discusión sobre objetos virtuales de aprendizaje: adaptabilidad, generatividad, escalabilidad y enfoques de diseño.

Los postulados del CAI (Computer Assisted Instruction) fueron recogidos en las décadas de 1980 y 1990 por los investigadores en sistemas 
tutoriales inteligentes (ITS 'Intelligent Tutoring System'), que buscaron proveer de más sustento conceptual al diseño de sistemas de instrucción y se enfocaron principalmente en generar entornos, en los que el conocimiento experto (del profesor) y sus estrategias fueran simuladas de manera eficiente por el sistema.

Tal vez sea L'Allier (1997) quien primero hable de "estructuras mínimas e independientes" para referirse a la idea de objetos de aprendizaje, distinguiendo en ellos tres partes: objetivo (qué se desea enseñar en ese objeto de aprendizaje), actividad de aprendizaje (los ejercicios e interactividad que desarrollará el alumno para reforzar los contenidos) y evaluación (puntuación obtenida en el desarrollo de la actividad).

Posteriormente, en 1999, el Institute of Electrical and Electronics Engineers (IEEE) los definiría como "como una entidad, digital o no digital que puede ser utilizada, reutilizada o referenciada durante el aprendizaje apoyado en tecnología”, y más tarde "como una entidad, digital o no digital que puede ser utilizada para el aprendizaje, la educación o el entrenamiento", mientras que Willey (1999) las semejaría con "pequeñas entidades instruccionales que pueden ser ensambladas en una estructura instruccional más grande", lo que se semeja mucho a la metáfora del mecano sugerida por Gerard (o a la del LEGO de Hodgins, 2004). Por su parte, la Nacional Learning Infrastructure Initiative (NLII) los asumió como "recursos digitales modulares, con identificadores únicos y descriptores, que pueden ser usados como soporte en el aprendizaje" (Educase, 2002). Polsani (2006) los describiría como "una unidad didáctica, autocontenida e independiente, predispuesta para su reutilización en múltiples contextos instruccionales".

Con esta multitud de visiones sobre los objetos de aprendizaje, el Ministerio de Educación Nacional de Colombia convocó a una mesa de trabajo en abril de 2006 que permitió llegar a acuerdos respecto de lo que se entendería en adelante como objeto de aprendizaje en el ámbito nacional y su diferenciación con otros recursos digitales usados en la educación soportada por tecnología.

De esta reunión, abordando el tema de manera estratégica pero a la vez ecléctica, surgen dos definiciones, basadas de algún modo en los trabajos de Cisco System: una respecto de los objetos de aprendizaje propiamente dichos y otra respecto 
de los objetos informativos. Así, para Colombia, "un objeto de aprendizaje es un conjunto de recursos digitales, autocontenible y reutilizable, con un propósito educativo y constituido por al menos tres componentes internos: contenidos, actividades de aprendizaje y elementos de contextualización. El objeto de aprendizaje debe tener una estructura de información externa (metadatos) que facilite su almacenamiento, identificación y recuperación". Mientras que "un objeto informativo es un conjunto de recursos digitales que puede ser utilizado en diversos contextos educativos y que posee una estructura de información externa (metadato) para facilitar su almacenamiento, identificación y recuperación" (Colombia, Ministerio de Educación Nacional [MEN], 2006). Será esta última definición de objeto de aprendizaje la que se tomará como base para el análisis del presente trabajo, pero agregando como nuevo elemento la evaluación, que en parecer del autor es necesaria para desarrollar dispositivos educativos que generen verdaderos procesos de aprendizaje.

Véase en este breve recuento histórico cómo el debate sobre los objetos de aprendizaje pareciera, desde sus inicios, estar más referido a las particularidades tecnológicas que sus propiedades didácticas; es decir, a su capacidad para comunicar información de manera estratégica, con el fin de propiciar la generación de conocimiento por parte de los individuos.

\section{Criterios en el diseño de objetos de aprendizaje}

En la presente investigación, una vez distinguido el debate en torno al concepto de objeto de aprendizaje, es necesario determinar ahora los criterios pedagógicos que, se supone, respaldarían el diseño de objetos de aprendizaje de corte constructivista $y$ orientados a un enfoque de competencias, dos de las tendencias que impulsa el Politécnico Grancolombiano como parte de su modelo educativo.

Desde el enfoque de competencias ha venido gestándose todo un movimiento de diseño didáctico que busca desarrollar unidades con capacidad de movilizar integralmente el saber conocer, el saber hacer, el saber ser y el saber saber. Para esto hay un acuerdo generalizado en que el conductismo o cognitivismo por sí solos resultan insuficientes, por lo que pareciera emerger una propuesta más cercana al uso de elementos propios del constructivismo, en el que mediante estrategias de enseñanza-aprendizaje 
(casos, proyectos, problemas, escenarios, simulación...) se busca aterrizar en la práctica los conocimientos adquiridos.

Este enfoque trata los problemas reales que enfrentan las profesiones como punto de partida de un currículo. Sus características principales son las siguientes: la utilización de materiales que simulan o tratan situaciones de la vida real, la disponibilidad de recursos para permitir a los educandos aclarar los problemas y enfrentarlos, el trabajo cooperativo de los educandos, el tratamiento de los problemas uno a la vez, diferente de los cursos basados en materias, donde se trata de hacer muchas cosas al tiempo.

Entonces el diseño instruccional para un enfoque de competencias

puede ser descrito esencialmente como un enfoque sistémico al diseño y desarrollo de instancias educativas que ayuden a los estudiantes a aprender. Como proceso se refiere a la traducción de principios generales de aprendizaje e instrucción en planes para el desarrollo de medios de aprendizaje. Es un proceso complejo, pero que es creativo, activo e interactivo (Hinrichsen, 2003).
Respecto de los materiales educativos, se plantea que deben ser ante todo modelo y guía de cómo hacer, guiar y orientar el aprendizaje proporcionando las condiciones necesarias para que se logre; seleccionar, implementar y presentar actividades; ayudar a ejecutar las actividades y constatar el nivel de aprendizaje; plantear situaciones desconcertantes, sin una solución obvia, que estimule la acción del alumno; deben adecuarse a las características del aprendizaje y a las etapas del desarrollo cognoscitivo del usuario; promueven una atmósfera de reciprocidad, de respeto y autoconfianza dando oportunidad para el aprendizaje autoestructurante de los alumnos, principalmente mediante la enseñanza indirecta y del planteamiento de problemas y conflictos cognitivos.

Desde el construtivismo. Para Mergel (1998), en el diseño desde una aproximación constructivista,

se requiere que el diseñador produzca estrategias y materiales de naturaleza mucho más facilitadora que prescriptiva. Los contenidos no se especifican, la dirección es determinada por el que aprende y la evaluación es mucho más subjetiva ya que no depende de criterios cuantitativos 
específicos, pero en su lugar se evalúan los procesos y el aprendiz realiza autoevaluaciones.

En este mismo sentido, Jonassen (1994) plantea que la construcción de conocimientos propuestos podrían facilitarse mediante un ambiente de aprendizaje que proporcione múltiples representaciones de la realidad, evite sobresimplificaciones, dé la instrucción por la representación de la complejidad natural del mundo, realice actividades reales auténticas -que estén contextualizadas-, proporcione un mundo real, ambientes de aprendizaje basados en casos, en lugar de instrucciones secuenciales predeterminadas, refuerce la práctica de la reflexión, faculte contextos y conocimientos dependientes de la construcción y soporte la construcción colaborativa de conocimientos a través de la negociación social.

Desde la comunicación del conocimiento. La relación comunicacióneducación ha estado determinada por la interdependencia teórica entre uno y otro campo de estudio. Aunque la reflexión sobre la educación es históricamente anterior a las teorías de la información que dieron cuenta de las primeras visiones del tema comunicativo, los diversos modelos pedagógicos han establecido, de una u otra manera, el papel que la interacción comunicativa desempeña en su interior, así no todos lo hayan reconocido explícitamente. Esto plantea, necesariamente, que del uso que se haga de la comunicación dependerá, en buena medida, el tipo de proceso de enseñanza-aprendizaje que se genere. Así es como una interacción básica, unidireccional, favorecerá un ambiente conductista, mientras que una abierta, multidireccional, acrecentará las posibilidades de la construcción colectiva de conocimiento.

Por tanto, es mediante el lenguaje que los individuos tienen la posibilidad de dar sentido (significar) a la realidad: en primera instancia procesando información proveniente del entorno de manera interna y luego objetivándola, tras la reflexión, en productos materiales o discursivos que pondrán en común con el fin confrontar las visiones propias con las de otros. Lenguaje que, concebido como capacidad biológica del individuo, como fluido simbólico que consiente y da sentido a la comunicación, es el que permite la socialización del conocimiento y, por tanto, la construcción de cultura mediante la objetivación de las experiencias tanto informales como formales. Las indagaciones 
psicogenéticas de Vygotsky (1995) han revelado el papel capital del lenguaje en el desarrollo de las facultades cognoscitivas:

el desarrollo del pensamiento está determinado por el lenguaje... El desarrollo de la lógica es una función directa del lenguaje socializado... El crecimiento intelectual depende del dominio de los mediadores sociales del pensamiento, esto es, del dominio de las palabras. El lenguaje es la herramienta del pensamiento (Kaplún, 1992).

Esto es válido tanto para la educación presencial como a distancia, o a la mediada por objetos de aprendizaje; lo que cambia, por supuesto, es el vehículo mediador del mensaje educativo.

En la educación a distancia, se han hecho numerosos aportes a la conceptualización del diseño de materiales educativos y las características comunicativo-didácticas cuando están orientados a procesos de autoestudio, siendo un ejemplo claro el de la guía didáctica. Si se retoman los conceptos que diversos autores han elaborado o retomado sobre el tema (García, 2001; Contreras, 2003), podemos definir la guía didáctica como un mediador pedagógico cuya labor principal es facilitar al estudiante la comprensión de los contenidos de una unidad de aprendizaje, en un proceso de estudio que él adelanta de manera autónoma, orientándolo sobre qué estudiar, cómo hacerlo, en qué ambientes y momentos, en qué contexto de aplicación y bajo cuáles criterios de evaluación; todo con la finalidad de lograr en el estudiante el desarrollo efectivo de las competencias planeadas. En este punto toma principal relevancia la teorías que Holmberg (1995) desarrolló en 1985 sobre la comunicación didáctica guiada, orientada a la interacción remota entre estudiantes y docentes, en la que los estudiantes establecen un diálogo simulado con el docente a través de su relación directa con los materiales de estudio y una comunicación real mediante el correo postal y el teléfono (ahora por chat, correo electrónico, foros, redes sociales).

Retomando a Holmberg, García (2001) diferencia entre el diálogo real y el simulado. Respecto del diálogo simulado, plantea que se da cuando

el interés de una institución que enseña es el de que se produzca aprendizaje en el receptor del proceso. Pero este aprendizaje ha de 
permitir al estudiante ser protagonista en cuanto al tiempo, espacio y ritmo de aprender, es decir, el proceso de enseñanza diseñado por la institución propicia el aprendizaje flexible del estudiante.

El diálogo didáctico simulado basa entonces su fundamento en la comunicación a través de medios como vehículo mediante el cual se produce el intercambio de sentidos necesario para que se produzca conocimiento en una relación de enseñanza-aprendizaje a distancia. Son los contenidos, en forma de materiales didácticos y distribuidos en diversos formatos, los que hacen flexible el aprendizaje, al facilitar los procesos de autoestudio. Dichos materiales deberán ser diseñados teniendo en cuenta diversos factores y las metodologías más adecuadas para el área disciplinar en la que se busca formar.

Entonces, con el fin de operativizar el diálogo didáctico simulado en el diseño de objetos de aprendizaje constructivistas, se haría necesario construir un material educativo que siendo programado, adecuado, preciso, actual, integral e integrador conduzca de manera efectiva el aprendizaje del alumno. Para esto, deberá ser coherente con el área disciplinar en la que se desea formar; predisponer una atmósfera de estudio agradable y cercana; partir de la información y los conocimientos previos del alumno para situarlo ante los nuevos contenidos; introducir de manera sencilla pero académica tanto los saberes por abordar como los métodos y las técnicas para alcanzarlos y convertirlos en habilidades, actitudes y valores reales; explicitar el papel y la importancia de los materiales de estudio básicos y complementarios; aludir a la afectividad del alumno para propiciar procesos de reflexión axiológica y ética; promover la reflexión crítica; propiciar la aplicación de saberes en ejercicios prácticos, cercanos y pertinentes, y generar procesos de reflexión de la práctica y del aprendizaje.

\section{Resultados}

En general, de la revisión de literatura se extrae que el diseño de todo objeto de aprendizaje que pretenda basarse en pedagogías activas y en un enfoque de competencias debería caracterizarse por una estructura didáctica que incluya los siguientes elementos:

- Metas de aprendizaje: el objeto de aprendizaje define metas 
de aprendizaje en términos de objetivos o competencias por alcanzar.

- Construcción de conocimiento: el diseño didáctico facilita el intercambio de sentidos entre aprendiz y contenidos —básicos y complementarios-, lo cual permite la negociación interna de saberes y la construcción de conocimiento aplicado.

- Soporte de aprendizaje: el objeto de aprendizaje contextualiza, orienta, aclara, ejemplifica y modela el aprendizaje cuestionando al aprendiz y facilitando la conexión de conocimientos presentes con conocimientos previos y futuros.

- Características del entorno: el objeto de aprendizaje es un ambiente programado, adaptado a las necesidades del aprendiz, pertinente, legible, coherente con las metas de aprendizaje propuestas y que se basta a sí mismo para alcanzarlas (autónomo).

- Escenario: el objeto de aprendizaje media la realidad y promueve su exploración mediante la planificación, secuenciada o flexible, de problemas, casos, simulaciones (entre otros), presentando contenidos programados y disponiendo herramientas de interacción y comunicación que permitan una acción reflexionada.

- Interactividad: la interactividad orienta al aprendiz en su accionar con el sistema, simula el diálogo didáctico, corrige seudoconceptos e indica los aciertos.

- Actividades de aprendizaje: las actividades buscan movilizar los aprendizajes hacia una práctica que permita solucionar problemas, comprender o construir productos y procesos generalizables.

- Evaluación: la evaluación está basada en criterios, orientada al mejoramiento y promueve el saber saber.

Todos los elementos mencionados deben estar presentes e integrarse de manera creativa y flexible para que el objeto de aprendizaje, más allá de informar, cumpla la función educativa para la que fue diseñado. Estos aspectos, no obstante, no difieren sino que complementan todos aquellos tecnológicos, ya mencionados arriba, orientados hacia otros aspectos, como la posibilidad de recuperación, uso, reuso, adaptación, interoperabilidad 
y escalabilidad de este tipo de materiales educativos. Es decir, como complemento de los criterios tecnológicos estos, más de carácter didáctico, permitirán acercarse al desarrollo de actividades educativas significativas para los estudiantes.

Respecto de la encuesta conducida a los docentes de la Facultad, que versaba sobre el uso y percepción de las tecnologías de la información y la comunicación en el entorno personal y en la educación, pueden extraerse las siguientes conclusiones:

1) Los docentes tienen acceso a múltiples tecnologías: el total de profesores manifestó tener acceso a un computador (portátil o de escritorio) para el desarrollo de sus actividades académicas; todos cuentan con dispositivo de telefonía celular, la mitad teléfonos inteligentes, y $14 \%$ dijo tener una tableta digital; de estos se conectan a internet principalmente mediante el computador y el teléfono inteligente, y lo hacen casi siempre desde el trabajo ( $80 \%$ ).

A estos dispositivos los profesores les otorgaron un grado de utilidad para la educación (sobre 5 puntos) de 4,9 al computador, de 3.4 a la televisión, de 2.9 al teléfono inteligente y de 1.4 a la tableta digital.
Paradójicamente, cuando se les pidió que atribuyeran el potencial de uso educativo comparado con otros posibles usos de estos dispositivos, los profesores le otorgaron la más baja calificación a la posibilidad educativa de los computadores (7\%), en comparación con investigar (43\%) e informarse (36\%). En este mismo sentido, el potencial educativo atribuido a los teléfonos inteligentes $y$ las tabletas digitales se reduce a cero, por debajo de las opciones comunicarse y entretenerse. Estas respuestas evidencian la percepción mayoritaria de que los dispositivos informáticos no poseen en sí mismos la capacidad de servir de entorno para el aprendizaje autónomo, sino más bien como espacios de consulta de información que se aprovecharán posteriormente en el aula.

El aprovechamiento de internet en la educación está dado, en gran medida, por la frecuencia de uso de determinadas aplicaciones y plataformas como apoyo. Respecto de este punto los profesores afirmaron que las plataformas más utilizadas en sus clases eran la de educación virtual (siempre $14 \%$, frecuentemente $43 \%$ y ocasionalmente $38 \%$ ), seguida de las de búsqueda y portales de información (siempre 14\%, frecuentemente 
$38 \%$ y ocasionalmente $14 \%$ ), portales de video tipo YouTube (siempre $0 \%$, frecuentemente $19 \%$ y ocasionalmente $62 \%$ ) y aplicaciones para el desarrollo de actividades en línea - creación de gráficos, editores, etc.(siempre $0 \%$, frecuentemente $19 \%$ y ocasionalmente $62 \%$ ). Quedan por debajo los blogs y las redes sociales como Facebook y Twitter que se usan ocasionalmente $(43,19$ y $5 \%$, respectivamente). Estos indicadores, si bien evidencian que existe un fuerte impacto de la política institucional de uso de la plataforma Moodle como apoyo a la educación presencial y de la implementación de televisores en cada aula, reflejan también que aún es necesario trabajar en el conocimiento de las potencialidades que tienen las diversas plataformas y cómo integrarlas en los procesos educativos de manera efectiva; pues estos datos refuerzan la percepción de que el principal uso que se da a internet es, por ahora, como repositorio de información, para hacer búsquedas, consultar información o ver videos de apoyo.

Finalmente, la encuesta interrogó a los docentes por el tipo de recursos que usaba en las aulas virtuales asociadas a sus cursos presenciales. El $100 \%$ afirmó poner documentos para lectura, $53 \%$ imágenes, $37 \%$ presentaciones, $32 \%$ actividades en línea con las herramientas de la plataforma, $26 \%$ videos o enlaces a ellos, $8 \%$ audios y solo $6 \%$ dijo integrar objetos de aprendizaje o interactivos de carácter educativo. Estos resultados indicarían que el uso de las aulas virtuales, más que entornos de interacción y desarrollo de actividades educativas, se da como repositorio de recursos educativos de carácter informativo.

Por otra parte, los datos arrojados por el análisis de las didácticas y los recursos digitales sugeridos o implementados en las aulas virtuales como apoyo a la presencialidad de los profesores de la Facultad, evidenciaron multiplicidad de estrategias y técnicas didácticas, algunas de corte tradicional, otras reconocidas por su uso en diversas universidades y disciplinas, pero también otras particulares de los programas de comunicación social, periodismo, mercadeo, medios audiovisuales, publicidad y diseño.

En este sentido, del total de aulas virtuales analizadas, en las que se evidenció la aplicación de más de una estrategia o técnica didáctica en cada una, las más usadas son en orden de importancia: el taller (14.8\%) que busca principalmente la aplicación de lo visto en clase o en 
la documentación, el método de casos (6.3\%), el ensayo, el método de proyectos, las exposiciones de estudiantes y los controles de lectura (4.7\%). Asimismo emergen didácticas particulares de las disciplinas analizadas, como creación artística, producción audiovisual, análisis de contenido (3.9\%); creación de guiones y formatos audiovisuales, producción para radio, investigación periodística $(3,1 \%)$; apreciación audiovisual, diseño de gráficos, búsqueda de ejemplificación y analogías, matrices de análisis de productos de comunicación, visitas de observación (2.3\%); creación de campañas, planes y estrategias de comunicación, debates, juegos de roles, mesas redondas y redacción periodística (1.6\%); análisis de discurso, creación de blogs, producción de animación, escritura libre, exploración de campo, investigación documental portafolio educativo, tutoriales y objetos de aprendizaje $(0.8 \%)$. De estas didácticas quedan excluidas metodologías reconocidas por su efectividad educativa como el aprendizaje colaborativo, el aprendizaje por pares, los clubes de aprendizaje, el método de problemas, las simulaciones y los WebQuest.

$\mathrm{Al}$ revisar la propuesta instruccional, la secuenciación y las formas de evaluación de estas propuestas didácticas en las aulas virtuales de los profesores, se hizo evidente que la mayoría de los cursos dan informaciones generales de lo que se busca y no explicitan, o lo hacen como una suma de productos, los criterios de evaluación. Lo que indica que las didácticas tienden a usarse de manera tradicional y poco estratégica. En este sentido, es claro que, si bien hay una variedad importante de didácticas, hace falta preparación por parte de los docentes para que diseñen actividades educativas que integren las metas de aprendizaje, los contenidos y la evaluación de manera coherente; en especial esta última, ya que se evidencian falencias en la determinación de métodos y estrategias evaluativas. El 65.4\% de los cursos carece de explicitación de criterios evaluativos, $17.7 \%$ tiene alguna especie de lista de criterios generales, $4.6 \%$ una escala de evaluación, $3.1 \%$ evaluación por cuestionarios y solo $3.1 \%$ tiene rúbricas de evaluación claramente diseñadas.

Esta tendencia se observa también en el tipo de recursos digitales implementados en las aulas virtuales, de los que solo $3.9 \%$ podría estar dentro de los parámetros aquí analizados como objetos de aprendizaje; 
se da pues prelación en los cursos a lecturas planas (100\%), imágenes (59\%) - la mayoría con una función estética- video (56.9\%), diapositivas $(31.4 \%)$ - en general diseñadas por los profesores-, videotutoriales (5.9\%) y enlaces a sitios web complementarios (2\%).

La situación es similar para de uso de la plataforma educativa, de la que no se explotan sus posibilidades para el diseño de las actividades y la gestión del conocimiento. Así, las principales herramientas que se usan son las siguientes: la de subida de archivos para entrega de trabajos (47.4\%), los foros de discusión (26.3\%) - muchas veces usados para entrega de trabajos más que para generar debates- y los cuestionarios (8.8\%) —-más enfocados en el desarrollo de exámenes de control de lectura-. Un $17.5 \%$ de las aulas virtuales no tiene ningún tipo de herramienta implementada, lo que sugiere que se usan como depósito de información, y no se encontró evidencia de uso de otras herramientas que podrían ayudar a desarrollar actividades educativas, como los chats, los glosarios, los wikis, las lecciones, los talleres y paquetes SCORM.

\section{Conclusiones}

Los resultados de la presente investigación, analizados de manera integral, permiten establecer un marco para el diseño de objetos de aprendizaje orientados a los programas académicos de la Facultad de Mercadeo, Comunicación y Artes del Politécnico Grancolombiano. Si bien los resultados pueden ser útiles para otras instituciones educativas, es claro que el estudio adelantado refleja algunas particularidades que deberían analizarse en cada contexto.

Para los propósitos de la investigación, se logra el objetivo de contar con algunos criterios que orienten el diseño de materiales educativos digitales para los programas de la Facultad. Por un lado, unos criterios de diseño instruccional generales, resultado de la revisión de literatura, orientados a la construcción de objetos de aprendizaje para metodologías activas y por competencias; por otro, un listado de didácticas particulares para los programas académicos de la Facultad que, aunque hay que explicitar con detalle para convertirlas en metodologías consistentes, son insumo para orientar un diseño alineado con las formas en enseñar $y$ aprender conceptos y destrezas propios de las disciplinas involucradas. Finalmente, 
queda un diagnóstico respecto del uso de tecnologías de información y comunicación como apoyo a la educación por parte de los profesores de la Facultad, que permitirá desarrollar acciones para mejorar aquellos aspectos señalados como deficientes.

Como colofón, quedan esbozadas algunas líneas de acción investigativa que pueden ayudar a validar los resultados y ampliar los alcances de la presente investigación. Por un lado, es necesario construir objetos de aprendizaje basados en los criterios aquí definidos y verificar su eficacia educativa; por otro, es importante profundizar en el uso de las didácticas generales y particulares que aquí emergieron, para sistematizarlas y explicitarlas con miras a hacer de ellas técnicas y estrategias reproducibles en la formación de estudiantes de los campos disciplinares involucrados tanto para el Politécnico Grancolombiano como para otras instituciones educativas.

\section{Referencias bibliográficas}

Atkinson, R. C. y Wilson, H. A. (1969). Computer-assisted instruction: a book of readings. Nueva York: Academic Press.
Colombia, Ministerio de Educación Nacional (2006). Mesa de trabajo: desarrollo de contenidos-objetos de aprendizaje. Memorias.

Contreras Lara, M. E. (2003). Propuesta para la elaboración de guías didácticas en programas a distancia. En Simposio Virtual Somece. Recuperado de http://www.somece.org.mx/ virtual2003/ponencias/contenidos/ guiasdidacticas/guiasdidacticas.htm Educase (2002). Nacional Learning Infrastructure Initiative (NLII). Recuperado de http://www.educause. edu/nlii/

García Aretio, L. (2001). La educación a distancia. De la teoría a la práctica. Barcelona: Ariel educación.

Gerard, R. W. (1969). Shaping the mind: computers in education. En R. C. Atkinson y H. A. Wilson, Computer-assisted instruction: a book of readings. Nueva York: Academic Press.

Gibbons, A. S., Nelson, J. y Richards, R. (2000). The nature and origin of instructional objects. En The instructional use of learning objects. D. A. Wiyle. Recuperado de http://www.reusability.org/read/chapters/gibbons.doc 
Hinrichsen, C. (2003). Educación del diseño basada en competencias: un aporte a la competitividad. Ed. DuocUC, Pontificia Universidad Católica de Chile. Recuperado de http://www. duoc.cl/proyecto-diseno/15.pdf

Hodgins, W. (2004). Into the future of learning objects. In Workshop on e-learning Objects \& Systems. Recuperado de http://grove.ufl.edu/ pgl/ events/Hodgins/future_lo.pdf

Holmberg, B. (1995). The evolution of the character and practice of distance education. Open Learning, 10(2), 47-53.

IEEE. History of IEEE. En: https:// www.ieee.org/about/ieee_history. html

Jonassen, D. H. (1994). Thinking technology: toward a constructivist design model. Educational Technology, 34(4), 34-37.

Kaplún, M. (1992). A la educación por la comunicación: la práctica de la comunicación educativa. Unesco/ Orealc.

L'Allier, J. J. (1997). Frame of Reference: NETg's Map to the products, their structure and core beliefs. Research and Development-NETg. Retrieved June, 25, 2013.

Mergel, B. (1998). Diseño instruccional y teoría del aprendizaje. Universidad de Saskatchewan, Canadá. Recuperado de http://etad.usask. ca/802papers/mergel/espanol.pdf

Polsani, P. R. (2006). Use and abuse of reusable learning objects. Journal of Digital Information, 3(4).

Vanegas, V. P. P. (1999). La guía didáctica, componentes estructurales. Cuernavaca, México: Universidad Autónoma del Estado de Morelos/Dirección de Educación a Distancia.

Vygotsky, L. S. (1995). Pensamiento y lenguaje. Barcelona: Paidós.

Wiley, D. A. (1999). The post-LEGO learning object. Recuperado de http://cmap.upb.edu.co/servlet/SBReadResourceServlet? rid=1146063082796_1162237027_9479 